\title{
Os efeitos do treinamento neurológico nas funções motoras de pacientes com isquemia cerebral
}

\author{
The effects of neurological training on the motor functions \\ of patients with cerebral ischemia
}

\author{
Los efectos del entrenamiento neurológico sobre las \\ funciones motrices de pacientes com isquemia cerebral
}

\begin{abstract}
Thálys Jair Melo Alves ${ }^{1}$, Aristarco de Pinho Ferreira Júnior ${ }^{1}$, Camila Monteiro Agostini ${ }^{1}$, Gustavo Felipe de Assis ${ }^{1}$, João Victor Lopes Campos $^{1}$, Ubiratan Rodrigues da Silva ${ }^{1}$, Laila Cristina Moreira Damázio ${ }^{2}$

1.Acadêmicos do Curso de Medicina da Universidade Federal de São João del-Rei, Campus Dom Bosco, Departamento de Medicina. São João del-Rei-MG, Brasil.

7.Fisioterapeuta. Professora do Curso de Medicina da Universidade Federal de São João del-Rei, Campus Dom Bosco, Departamento de Medicina. São João del-Rei-MG, Brasil.
\end{abstract}

\begin{abstract}
Resumo
Introdução. O Acidente Vascular Cerebral (AVC) é a doença que mais causa incapacidade funcional no mundo. Objetivo. Investigar os efeitos do treinamento neurológico funcional no desempenho motor e qualidade de vida de pacientes com sequelas de AVC e avaliar a saúde e a qualidade de vida de seus cuidadores. Método. Foram reabilitados oito pacientes, os quais realizaram exercícios funcionais, simples e dinâmicos todos os dias com uma média de 4 a 8 horas de atividades por dia, durante 6 dias por semana em 8 semanas. Para avaliação da funcionalidade dos pacientes foi utilizada a escala Fugl-Meyer e o questionário SF-36 para a qualidade de vida. Os dados das escalas antes e após o treinamento neurológico funcional foram analisados com o auxílio do programa estatístico GraphPrismPad 5.0, utilizando como teste estatístico o t-student pareado, considerando como nível de significância $p<0,05$. Resultados. Os dados da escala Fugl-Meyer, demonstraram diferenças na função motora dos membros inferiores e superiores do membro afetado, na coordenação/velocidade e no total da função motora. Na avaliação da qualidade de vida foi evidenciada diferença apenas para o domínio aspecto social. Os dados da escala SF-36 entre os cuidadores, não demonstrou diferença para nenhum domínio. Conclusão. A realização de treinamento neurológico funcional em pacientes com sequelas de AVC contribui na melhora da aquisição de competências motoras.
\end{abstract}

Unitermos. Terapia por Exercício; Acidente Vascular Cerebral; Movimento; Qualidade de Vida

\begin{abstract}
Introduction. Stroke is the disease that most causes functional disability in the world. Objective. To investigate the effects of functional neurological training on the motor performance and quality of life of patients with CVA sequelae and to assess the health and quality of life of their caregivers. Method. Eight patients were rehabilitated, who performed functional, simple, and dynamic exercises every day with an average of 4 to 8 hours of activities per day, during 6 days per week in 8 weeks. The Fugl-Meyer scale and the SF-36 questionnaire for quality of life were used to evaluate the patients' functionality. The data of the scales before and after the functional neurological training were analyzed with the aid of the statistical program GraphPrismPad 5.0, using the paired t-student as statistical test, considering as significance level $p<0.05$. Results. The Fugl-Meyer scale showed differences in motor function of the lower and upper limbs of the affected limb, in coordination/speed and in total motor function. In the evaluation of the quality of life was evidenced difference only for the social aspect domain. Data from the SF-36 scale among caregivers did not show a difference for any domain. Conclusion. The performance of functional neurological training in
\end{abstract}


patients with sequelae of stroke contributes to the improvement of the acquisition of motor skills.

Keywords. Exercise Therapy; Stroke; Movement; Quality of life

\section{Resumen}

Introducción. El Accidente Vascular Cerebral (AVC) es la enfermedad que más causa discapacidad funcional en el mundo. Objetivo. Investigar los efectos del entrenamiento neurológico funcional sobre el rendimiento motor y la calidad de vida de los pacientes con secuelas de accidente cerebrovascular y evaluar la salud y la calidad de vida de sus cuidadores. Método. Se rehabilitaron ocho pacientes, quienes realizaron ejercicios funcionales, simples y dinámicos todos los días con un promedio de 4 a 8 horas de actividades diarias, durante 6 días a la semana en 8 semanas. Para evaluar la funcionalidad de los pacientes se utilizó la escala de Fugl-Meyer y el cuestionario SF-36 de calidad de vida. Los datos de la escala antes y después del entrenamiento neurológico funcional se analizaron con la ayuda del programa estadístico GraphPrismPad 5.0, utilizando la prueba estadística t-student pareada, considerando un nivel de significancia de $p<0.05$. Resultados. Los datos de la escala de FuglMeyer mostraron diferencias en la función motora de los miembros inferiores y superiores del miembro afectado, en la coordinación / velocidad y en la función motora total. En la evaluación de la calidad de vida, se encontró una diferencia solo para el dominio del aspecto social. Los datos de la escala SF-36 entre los cuidadores no mostraron diferencias para ningún dominio. Conclusión. La realización del entrenamiento neurológico funcional en pacientes con secuelas de ictus contribuye a mejorar la adquisición de habilidades motoras.

Palabras clave. Terapia de ejercicio; Accidente vascular cerebral; Movimiento; Calidad de vida

Trabalho realizado na Universidade Federal de São João del-Rei, Campus Dom Bosco, Departamento de Medicina. São João del-Rei-MG, Brasil.

Conflito de interesse: não Recebido em: 11/12/2020

Aceito em: 11/06/2021

Endereço para correspondência: Laila CM Damázio. Departamento de Medicina, UFSJ. Praça Dom Helvetia 74. São João del-Rei-MG, Brasil. Cep 36301-160. Fone: (32) 3379-5572. Email: lailadamazio@ufsj.edu.br

\section{INTRODUÇÃO}

O acidente vascular cerebral (AVC) é uma anormalidade cerebral causada por alterações nas paredes dos vasos e pertence ao grupo de doenças cerebrovasculares ${ }^{1}$. De acordo com a Sociedade Brasileira de Doenças Cerebrovasculares (2015), o AVC é a doença com maior mortalidade no Brasil e a principal causa de incapacidade no mundo. A Organização Mundial de Saúde (OMS) estima que 6,7 milhões de pessoas morrem com AVC por ano².

Os principais fatores de risco para as doenças cerebrovasculares são a hipertensão arterial, dislipidemia, 
tabagismo, diabetes mellitus, dietas ricas em gorduras/colesterol, hipertrofia ventricular esquerda, fatores trombogênicos, sedentarismo, obesidade e consumo de álcool $^{3}$. Sendo que, os pacientes com história de AVC recente ( $<5$ anos) tem risco consideravelmente maior de sofrer um novo AVC no futuro 4 .

Os pacientes com AVC podem apresentar alterações motoras significativas como a diminuição ou perda súbita da força nos músculos da face, braço ou perna de um lado do corpo; instabilidade postural e desequilíbrio ${ }^{5}$. A fraqueza muscular é uma das alterações mais significativas após o AVC $^{6}$. No decorrer da evolução do quadro, o tônus tende a aumentar e a espasticidade, que é o aumento da resistência ao alongamento passivo, se instala?.

Muitos estudos têm aplicado intervenções que minimizem os riscos de quedas e melhoram a marcha dos pacientes com $\mathrm{AVC}^{8-11}$. Atualmente, vários grupos de pesquisa têm estudado novas alternativas de reabilitação de pacientes com AVC e a terapia neuromotora intensiva (TNMI) é uma denominação recente utilizada para se referir a protocolos terapêuticos com grande diversidade de recursos e que são desenvolvidos com mais de duas horas por dia de intervenção, por pelo menos, cinco dias na semana, durante mais de quatro semanas de reabilitação ${ }^{12,13}$.

O Treinamento em Reabilitação Neurológica Intensiva é um tipo de TNMI que apresenta um novo conceito de tratamento elaborado a partir de estudos científicos na área de reabilitação, treinamento profissional nacional e 
internacional e pesquisas clínicas observacionais durante os últimos 15 anos. Tem por objetivo melhorar a independência e a autonomia dos indivíduos que sofreram lesão e/ou disfunção no sistema nervoso central, e que podem ser submetidas ao treinamento intensivo em reabilitação ${ }^{14}$.

O sucesso na execução dessa intervenção terapêutica depende da participação ativa dos cuidadores para melhora da funcionalidade e qualidade de vida desses pacientes vítimas de AVC. Sabe-se que não só os pacientes, mas também seus cuidadores precisam de atenção e apoio profissional, pois, orientar os cuidadores quanto à saúde e atividades de vida diária traz benefícios à recuperação do paciente e ainda proporciona maior tranquilidade e qualidade de vida aos que desempenham a difícil tarefa de cuidar ${ }^{15}$.

Com isso, o objetivo do estudo foi investigar os efeitos do treinamento neurológico funcional no desempenho motor e qualidade de vida de pacientes com sequelas de AVC e avaliar a saúde e a qualidade de vida de seus cuidadores de um município no interior de Minas Gerais e região.

\section{MÉTODO}

O presente estudo apresenta delineamento experimental com abordagem quantitativa e foi realizado no período de maio a dezembro de 2017, em um município no interior de Minas Gerais/Brasil. A pesquisa foi executada em três Estratégias de Saúde da Família (ESF's) do município. 
O estudo foi submetido e aprovado pelo Comitê de Ética envolvendo Seres Humanos com número de CAAE de 61775316.0.0000.5545.

\section{Amostra}

Foram selecionados por conveniência 10 pacientes vítimas de AVC, registrados nas ESF's descritos anteriormente, que encontravam com limitações físicas em seus domicílios e aceitaram participar da pesquisa. Esses pacientes foram avaliados pelo médico colaborador da pesquisa para liberação para execução dos exercícios físicos. Desses 10 pacientes, 2 realizavam reabilitação com fisioterapia tradicional e foram retirados da pesquisa para não interferirem nos dados da pesquisa.

Dessa forma, foram excluídos da pesquisa os pacientes que não apresentaram sequelas do AVC; pacientes aos quais não apresentaram liberação médica para execução dos exercícios físicos; pacientes que não compreenderam as perguntas da escala SF-36, pacientes com afasia de compreensão; pacientes com problemas cardíacos graves; pacientes com doenças ortopédicas e reumáticas graves que impossibilitasse a realização dos exercícios e os pacientes que realizavam fisioterapia tradicional.

\section{Procedimentos}

O presente estudo utilizou as escalas de Fugl-Meyer e o questionário SF-36, respectivamente. A escala de avaliação de desempenho físico de Fugl-Meyer é um protocolo de 
avaliação do comprometimento motor que possui cinco dimensões, incluindo três aspectos do controle motor, como a amplitude de movimento articular, dor, sensibilidade, comprometimento motor da extremidade superior e inferior e equilíbrio. Cada item apresenta subitens que são pontuados em uma escala ordinal de 3 ( $0=$ nenhuma performance; 1=performance moderada; 2 =performance completa). O escore total alcançável é de 100 pontos. Cacho et al. ${ }^{16}$ propõe estratificar o comprometimento motor em quatro níveis: nível severo - corresponde as pontuações menores que 50, que representam pequena ou nenhuma movimentação voluntária dos membros afetados; nível marcante - com pontuação de 51 a 84; nível moderado - com pontuação entre 85 a 95, onde especificamente a função da mão pode estar altamente comprometida e o nível leve de comprometimento motor - quando a pontuação entre 96 a 99.

A qualidade de vida e o estado geral de saúde, dos pacientes e seus cuidadores foram avaliadas com auxílio da escala SF-36 que é um questionário composto de 36 itens que mensura a qualidade de vida em oito diferentes pontos. A SF-36 também é utilizada para avaliação do estado geral de saúde. Esta escala está entre os mais utilizados métodos de avaliação de qualidade de vida em estudos com pacientes. A estratificação do SF-36 é feita em oito diferentes escalas que incluem: percepções de saúde geral, funcionalidade física, limitações devidas às funcionalidades físicas, dores 
corporais, saúde mental geral, limitações devidas aos problemas emocionais, vitalidade e interações sociais ${ }^{17}$.

Para reabilitação dos pacientes com sequelas de AVC foi utilizado o treinamento neurológico funcional (treinamento neurológico intensivo adaptado) que é uma intervenção nova no tratamento dos pacientes e pode ser realizado entre 8 a 16 horas por dia, dependendo do tipo de abordagem e o desenvolvimento do paciente. A intervenção pode ser realizada seis dias por semana, no mínimo de quatro semanas ${ }^{14}$.

No início e no final da pesquisa o grupo foi avaliado pelos instrumentos (escala Fugl-Meyer e SF-36) descritos acima. Durante três dias consecutivos foram aplicadas as escalas sequencialmente, sendo aplicada uma escala a cada dia para não cansar o paciente. No terceiro dia os cuidadores também foram avaliados através da escala SF-36.

Para aplicação da escala SF-36, o paciente foi levado a uma sala com mesa e cadeira e foi munido de uma caneta, recebendo então as instruções para realização do questionário. Os examinadores foram devidamente treinados, no intuito de evitar interferência de resultados por heterogeneidade nos graus de instrução da população avaliada, segundo recomendado por Teixeira-Salmela et al6. Posteriormente, foram calculados os escores e foi realizada sua transformação em notas de oito domínios que variaram de zero a cem. Quando o paciente não conseguia manipular a caneta para responder a escala, o avaliador preenchia os itens da escala de acordo com a resposta do paciente. Os 
cuidadores dos pacientes também foram avaliados por esse instrumento.

Para a execução da reabilitação foi utilizado o treinamento neurológico funcional, onde os pacientes realizaram exercícios funcionais, simples e dinâmicos todos os dias. A reabilitação foi realizada com uma média de 4 a 8 horas de atividades por dia, durante seis dias por semana por oito semanas. Toda semana a equipe de pesquisa visitou o paciente e filmou a execução dos exercícios orientados. Os pesquisadores deixaram uma agenda de exercícios que foi ensinada para o paciente e o cuidador. Durante a semana, o cuidador supervisionou e orientou a execução dos exercícios da agenda. A agenda de exercícios foi simples, segura e motivadora. Os exercícios da agenda enfatizaram alongamento dos membros superiores, membros inferiores e tronco; fortalecimento muscular global e exercícios funcionais do dia a dia. A progressão dos exercícios foi semanal e a evolução do paciente foi o termômetro do terapeuta/pesquisador.

\section{Análise Estatística}

Os dados das escalas antes e após o treinamento neurológico funcional foram analisados com o auxílio do programa estatístico GraphPrismPad 5.0, utilizando como teste estatístico o t-student pareado, considerando como nível de significância $p<0,05$. 


\section{RESULTADOS}

As análises dos resultados obtidos na escala Fugl-Meyer aplicada nos membros inferiores dos oito participantes da pesquisa no início do tratamento e após as oito semanas de reabilitação demonstraram diferenças nas médias em todos os parâmetros avaliados nos membros, não afetados e afetados (Tabela 1 ).

No teste da extremidade inferior foi observada uma diferença na média do membro não afetado de 2,28 no escore da escala, antes e depois da intervenção, não demonstrando diferença $(p=0,1970)$. Já no membro afetado a diferença foi de $3,87(p=0,0381)$, indicando diferença nos tempos analisados, como demonstrado na Figura 1.

Tabela 1. Médias dos dados da Fugl-Meyer dos membros inferiores antes e depois da reabilitação, no membro não afetado (NA) e afetado (A).

\begin{tabular}{ccccccc}
\hline & \multicolumn{2}{c}{$\begin{array}{c}\text { Extremidade } \\
\text { superior }\end{array}$} & \multicolumn{2}{c}{ Coordenação/velocidade } & \multicolumn{2}{c}{$\begin{array}{c}\text { Total função } \\
\text { motora }\end{array}$} \\
\hline & NA & A & NA & A & NA & A \\
Antes & 23,1 & 11,38 & 4,375 & 1,375 & 27,5 & 11,38 \\
Depois & 25,38 & 15,25 & 5,25 & 4,25 & 30,63 & 19,5 \\
$\begin{array}{c}\text { Diferença média } \\
\text { antes e depois }\end{array}$ & 2,28 & $3,87^{* *}$ & 0,0875 & $2,875^{* *}$ & 3,13 & $8,12^{* *}$ \\
\hline \multicolumn{2}{r}{ *NA=Não afetado e A+afetado. ** Valor de $\mathrm{p}=0,05}$. & & & \\
\hline
\end{tabular}

Os resultados de Coordenação/Velocidade dos membros inferiores demonstraram diferença na média do membro não afetado $(p=0,1755)$. Sendo que, no membro afetado, a diferença encontrada foi de 2,875 ( $p=0,0071$; Figura 1). 
A análise do total da função motora dos membros inferiores demonstrou uma diferença média para o membro não afetado de 3,13 pontos $(p=0,1412)$, e para o membro afetado a diferença média foi de $8,12(p=0,0115$; Figura 1$)$.

MEMERO SADIO (A)
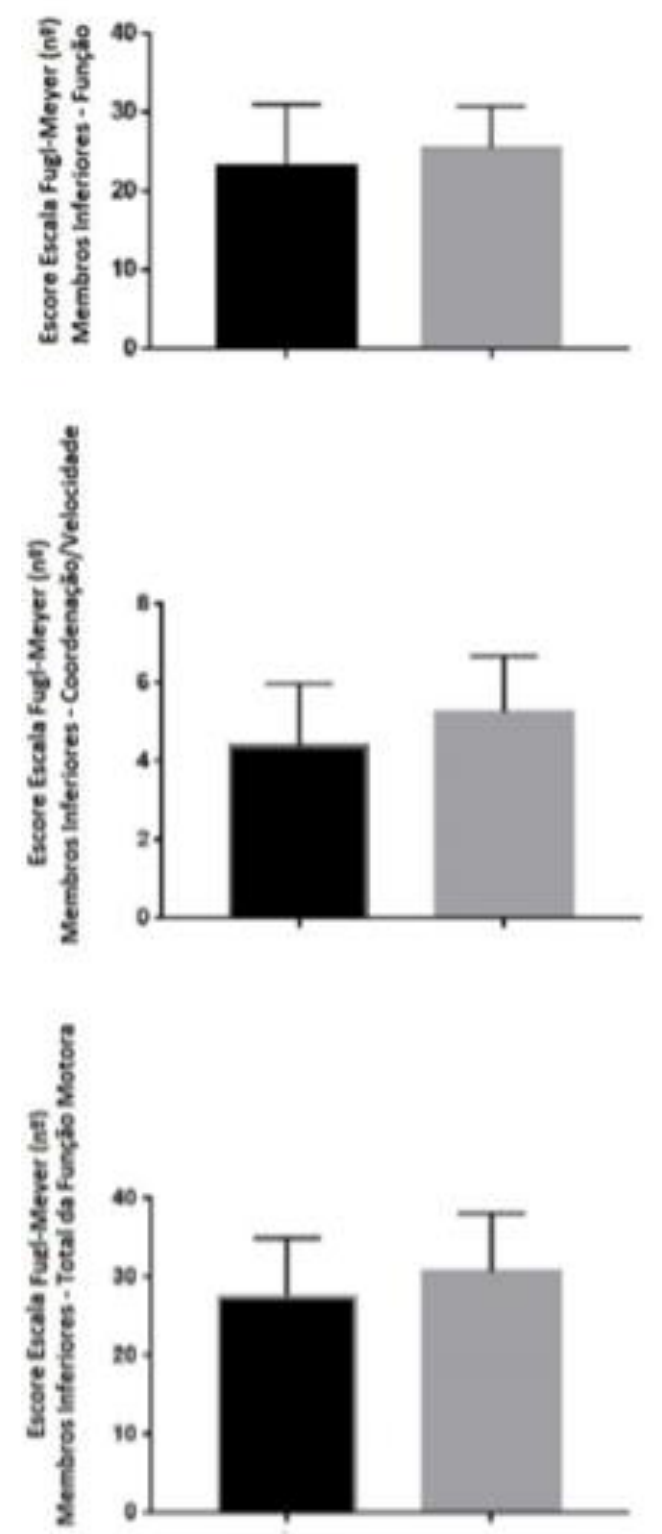

MEMERO AFETADO (B)
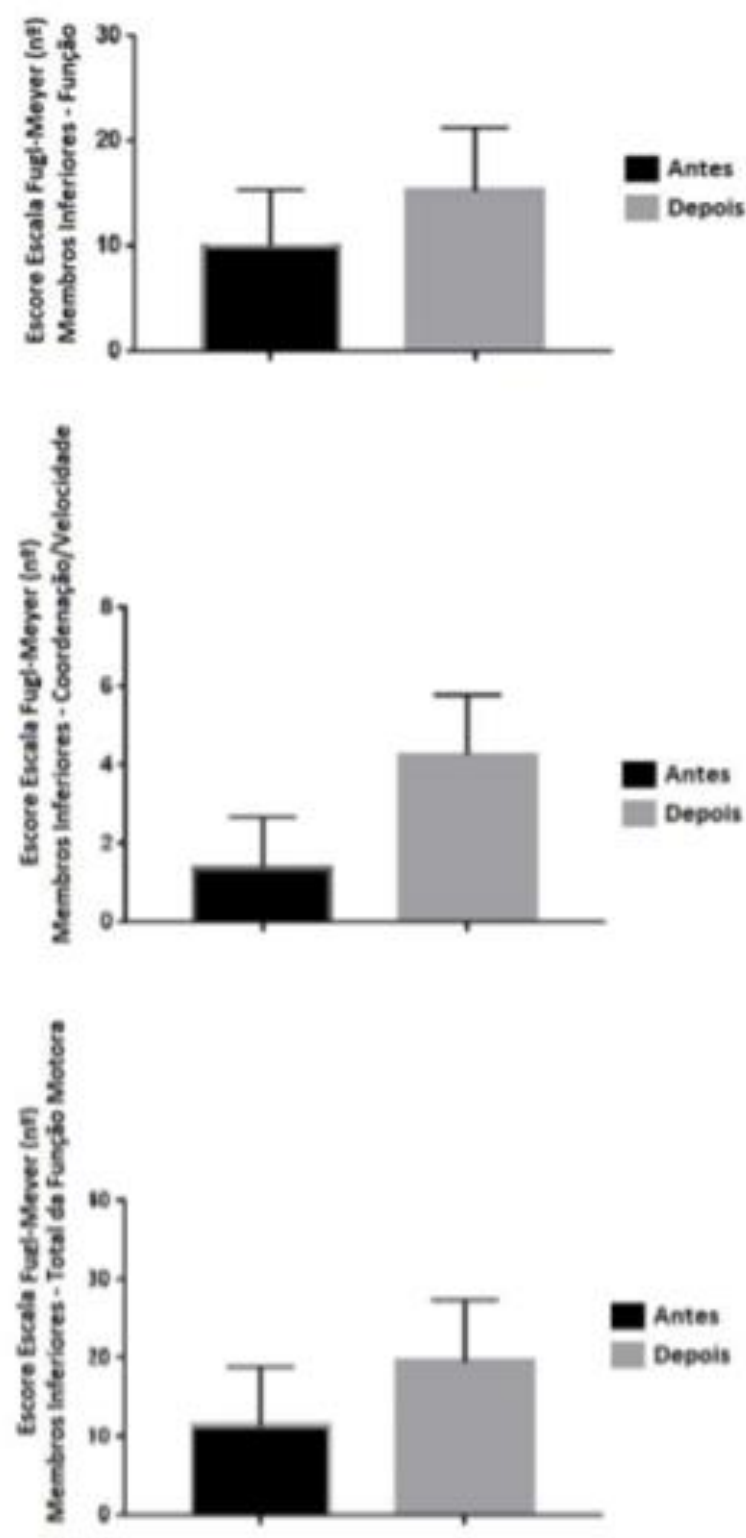

Figura 1. Dados dos escores da escala Fugl-Meyer dos membros inferiores em (A) membro sadio e (B) membro afetado pelo AVC. Dados da função motora, coordenação/velocidade e total da função motora (teste $t$-student, $p<0,05$ ). 
Os resultados obtidos na escala Fugl-Meyer aplicada nos membros superiores dos oito participantes da pesquisa no início do tratamento e após a reabilitação, demonstraram diferenças nas médias de todos os testes nos membros não afetados e afetados (Tabela 2), sendo que, no teste da extremidade superior notamos uma diferença da média no membro não afetado de 1,87 no escore da escala $(p=0,2207)$. No membro afetado a diferença foi de 2,15 $(p=0,3301)$.

Tabela 2. Médias dos dados da Fugl-Meyer dos membros superiores antes e depois da reabilitação, no membro não afetado (NA) e afetado (A).

\begin{tabular}{ccccccccccc}
\hline & \multicolumn{2}{c}{$\begin{array}{c}\text { Extremidade } \\
\text { superior }\end{array}$} & Punho & & Mão & & \multicolumn{2}{c}{$\begin{array}{c}\text { Coordenação } \\
\text { velocidade }\end{array}$} & $\begin{array}{c}\text { Total função } \\
\text { motora }\end{array}$ \\
& NA & A & NA & A & NA & A & NA & A & NA & A \\
Antes & 32,88 & 14,75 & 8,75 & 4,25 & 13,1 & 6,25 & 5,5 & 2,375 & 60,25 & 27,25 \\
Depois & 34,75 & 16,9 & 10 & 6,25 & 14 & 9,875 & 6 & 4,5 & 64,75 & 37,5 \\
$\begin{array}{c}\text { Diferença média } \\
\text { antes e depois }\end{array}$ & 1,87 & 2,15 & 1,25 & 2 & 0,9 & 3,625 & 0,5 & 2,125 & 4,5 & 10,25 \\
\hline
\end{tabular}

Os resultados da função motora do punho demonstraram diferença na média do membro não afetado de 1,25 ( $p=0,1705) ;$ enquanto, no membro afetado a diferença encontrada foi de $2(p=0,0811)$.

$\mathrm{Na}$ análise da função motora da mão foi encontrado diferença na média do escore da mão não afetada de 0,9 $(p=0,3506)$ e na mão afetada foi de 3,625 ( $p=0,0992 ;$

Figura 2). 
Nos resultados de Coordenação/Velocidade dos membros superiores foram encontramos diferença na média do membro afetado de 0,5 pontos $(p=0,2275)$ e no membro afetado, a diferença encontrada é de 2,125 ( $p=0,0011$; Figura 2).

Na análise do total da função motora dos membros superiores foi evidenciado uma diferença média para 0 membro não afetado de 4,5 ( $p=0,2163)$, sem diferença, e para o membro afetado a diferença média foi de 10,25 $(p=0,0659$; Figura 2).

Os resultados da avaliação da qualidade de vida de todos os pacientes com sequelas de AVC e seus cuidadores foram avaliados utilizando a escala de SF36, que apresenta oito domínios, sendo estes: capacidade funcional (CF), limitação por aspecto físico (LAF), dor (D), estado geral de saúde (EGS), vitalidade (V), aspecto social (AS), limitação por aspecto emocional (LAE) e saúde mental (SM). Para estes oito domínios, os valores médios dos itens LAF, D, EGS, $\mathrm{V}$, AS e SM foram superiores para os pacientes após a realização do treinamento neurológico funcional, no entanto, foi observada diferença $(p<0,05)$ apenas para o domínio AS (Figura 3).

Os dados da escala SF-36 entre os cuidadores, demonstrou alteração somente nos valores médios dos itens $C F, V$, AS, e LAE, onde foram superiores após o treinamento neurológico funcional dos pacientes, não sendo observada diferença para nenhum domínio (Figura 4). 

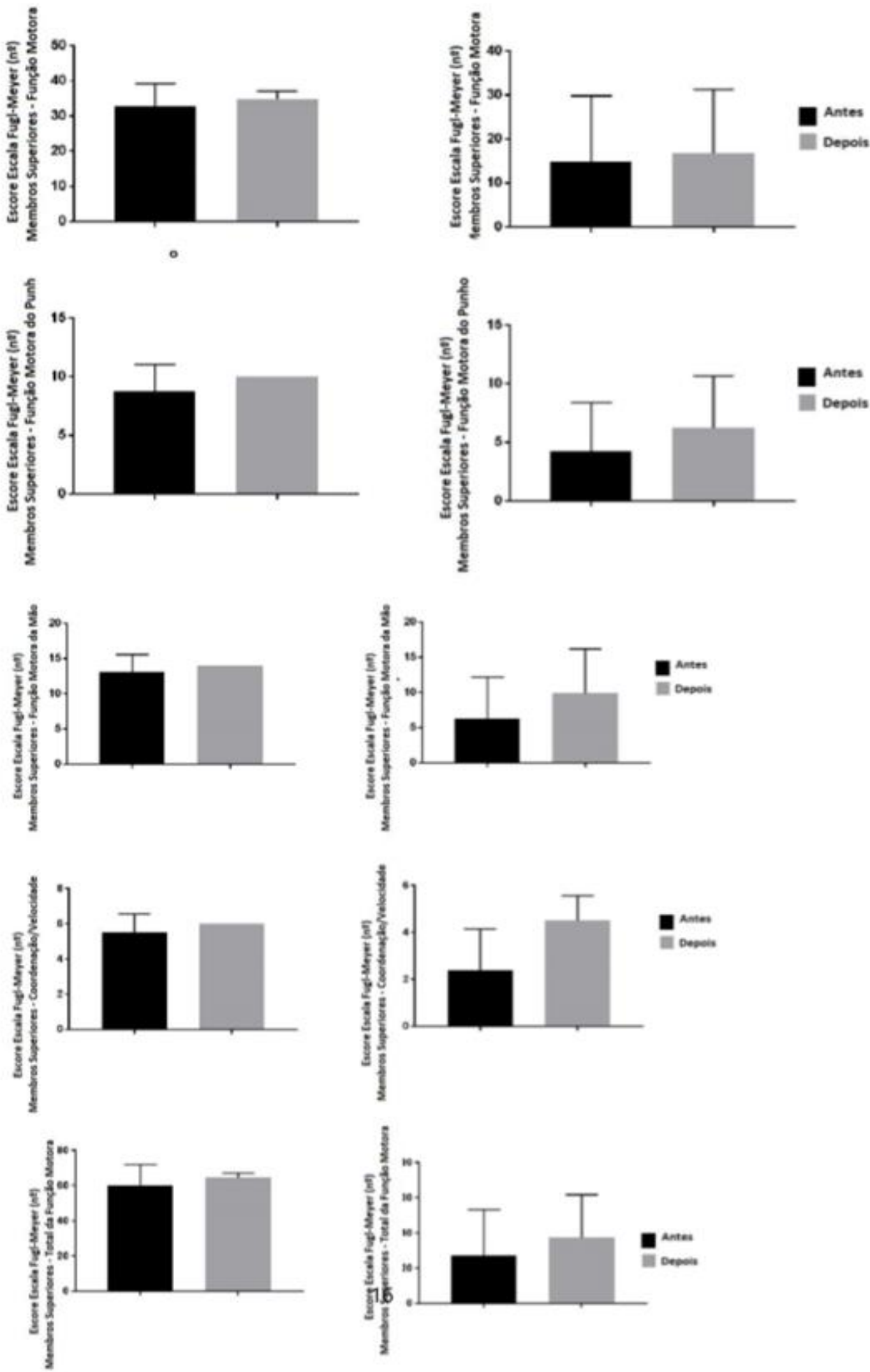

Figura 2. Dados dos escores da escala Fugl-Meyer dos membros superiores em (A) membro sadio e (B) membro afetado pelo AVC. Dados da função motora, coordenação/velocidade e total da função motora (teste $t$-student, $\mathrm{p}<0,05$ ). 


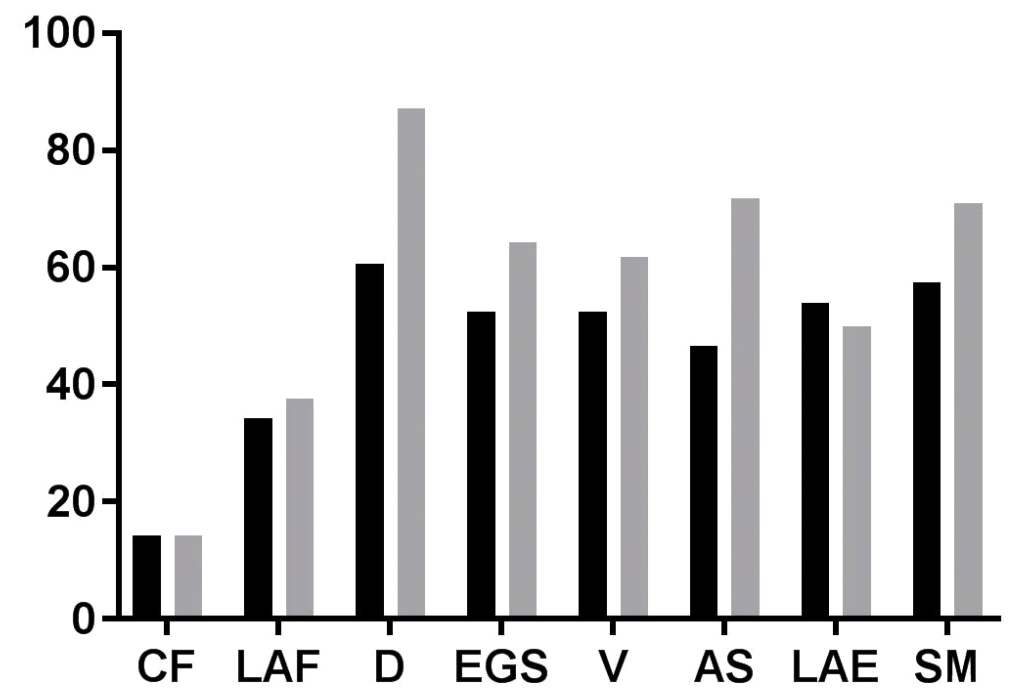

Figura 3. Dados médios da escala SF-36 entre os pacientes tratados antes e depois do treinamento neurológico funcional (teste $t$-student, $\mathrm{p}<0,05$ ).

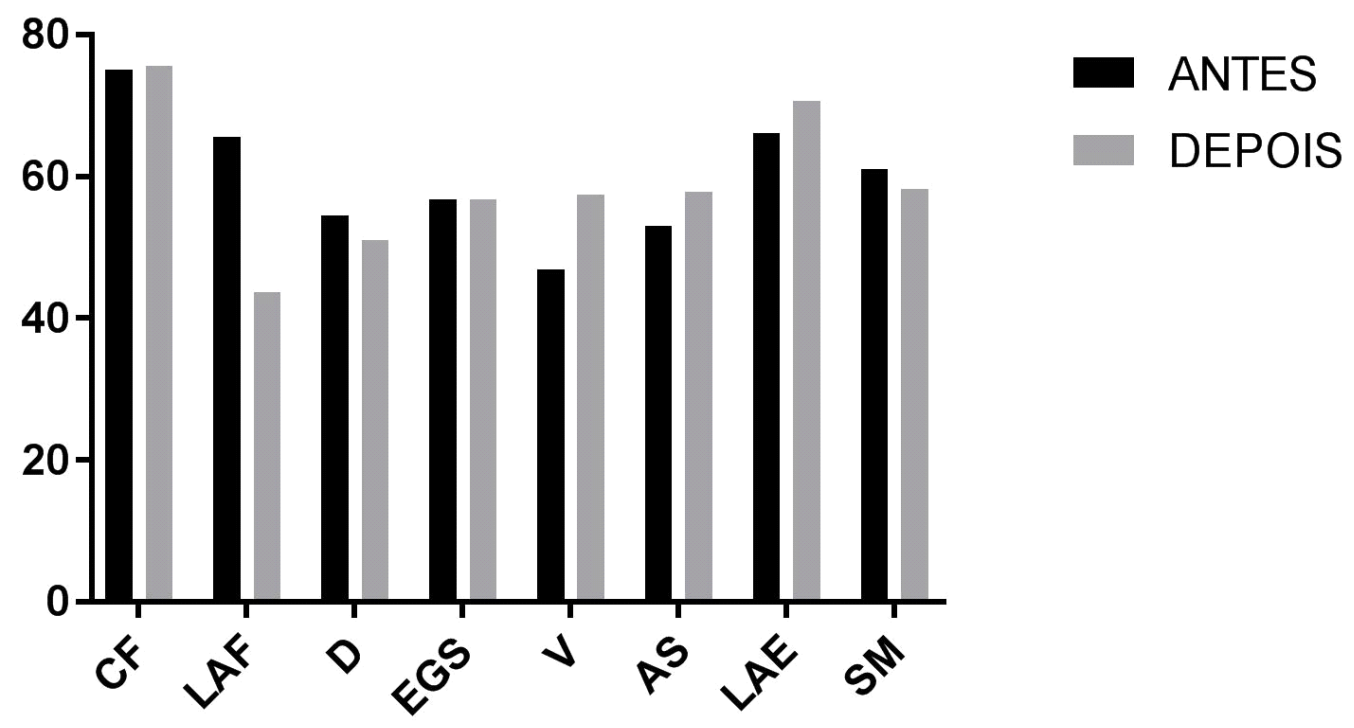

Figura 4. Dados médios da escala SF-36 entre os cuidadores antes e depois do treinamento neurológico funcional dos pacientes (teste $t$-student, $p<0,05$ ). 


\section{DISCUSSÃO}

Os resultados do presente estudo demonstraram que 0 treinamento neurológico funcional beneficiou as funções motoras dos pacientes com sequelas de AVC, sendo que, as funções motoras dos membros inferiores foram mais beneficiadas que as funções dos membros superiores. $O$ treinamento neurológico funcional é considerado como um TNMI, e esse treinamento beneficia o paciente com AVC melhorando sua funcionalidade e desempenho motor ${ }^{12}$.

O treinamento neurológico funcional melhora a funcionalidade dos pacientes com AVC possibilitando que os cuidadores ou familiares auxiliem em todo o processo da reabilitação ${ }^{14}$. Assim, o período de reabilitação começa a fazer parte da rotina do paciente acelerando o processo da sua recuperação.

Os dados desta pesquisa demonstraram que os ganhos funcionais foram melhores nos membros superiores em comparação com os membros inferiores dos pacientes. Este fato pode ser explicado num estudo onde foi sugerido que a recuperação de membros superiores tem piores resultados devido aos maiores déficits sensoriais que levam a um mecanismo de não aprendizado ${ }^{18}$.

Os resultados da qualidade de vida entre os pacientes demonstraram que ocorreu aumento em todos os parâmetros da escala SF-36 quando comparado o antes e depois do treinamento neurológico funcional. No entanto, só foi observada diferença no parâmetro relacionado ao Aspecto Social. O Aspecto Social da escala de qualidade de vida se 
refere à percepção dos indivíduos quanto à sua interação social, em meio familiar e ambiente de tratamento, de forma que foi possível observar melhora no relacionamento social dos pacientes em seu ambiente de convívio e a maior reintegração social em suas atividades de vida diária $(A V D \text { 's })^{19}$.

A avaliação da qualidade de vida dos cuidadores demonstrou que ocorreu aumento dos parâmetros: capacidade funcional, vitalidade, aspectos sociais e limitação por aspecto emocional, antes e depois da reabilitação dos pacientes. No entanto, não ocorreu diferença em nenhum dos aspectos da qualidade de vida. Esses dados podem ser explicados considerando o modelo de sobrecarga de trabalho dos cuidadores, além da sensação de pressão e responsabilidade para o cumprimento das tarefas que geram sentimentos estressores ao cuidador ${ }^{20}$.

A reabilitação do paciente melhora a independência funcional e gera satisfação e bem-estar ao paciente possibilitando a diminuição da dependência na execução das suas AVD's. No entanto, o cuidador ainda tem a responsabilidade de auxiliar uma pessoa com um grave comprometimento da saúde. Dessa forma, não foi possível observar ganhos significativos na qualidade de vida dos cuidadores. Esses dados corroboram com outro estudo onde também não foi identificada melhoras na qualidade de vida dos cuidadores dos pacientes com sequelas de AVC após reabilitação ${ }^{21}$. 
O treinamento neurológico funcional utilizado no presente estudo priorizou a melhora da independência funcional dos pacientes onde foi possível observar benefícios significativos no ganho de função motora geral entre os pacientes reabilitados. Acredita-se que 0 processo de neuroplasticidade cerebral $^{22}$ foi beneficiado com essa intervenção onde foi possível melhorar as habilidades motoras dos pacientes.

Com isso, o treinamento neurológico funcional demonstrou ser uma estratégia terapêutica eficiente na reabilitação dos pacientes com sequelas de AVC e que a intervenção poderá ser inserida no cotidiano dos pacientes e de seus cuidadores diariamente.

\section{CONCLUSÃO}

Conclui-se que a realização do treinamento neurológico funcional em pacientes com sequelas de AVC, com o auxílio dos seus cuidadores, contribuiu para melhora da função motora e qualidade de vida dos pacientes. Sendo que, foi evidenciado maiores ganhos na função motora do membro inferior em relação ao membro superior dos pacientes tratados.

\section{AGRADECIMENTOS}

Às Pró-Reitorias de Pesquisa e Pós-Graduação e Extensão da UFSJ/PROPE/PROEX, além do Órgão de Fomento de Minas Gerais, FAPEMIG e o Projeto 
PET/Saúde/GraduaSUS do Ministério da Saúde e Educação do Brasil.

\section{REFERÊNCIAS}

1.Brasileiro-Filho G. Bogliolo Patologia. Rio de Janeiro: Guanabara Koogan, 2011.

2.DATASUS 2016 (endereço na internet). Brasil: Ministério da Saúde, POrtal da Saúde (acessado em 2020). Disponível em: http://www2.datasus.gov.br/DATASUS/index.php?area=0203

3.Simão AF, Precoma DB, Andrade JP, Correa Filho H, Saraiva JFK, Oliveira GMM, et al. I Diretriz Brasileira de Prevenção Cardiovascular. Arq Bras Cardiol 2013;101:1-63.

https://doi.org/10.5935/abc.20135012

4. Howard G, Kissela BM, Kleindorfer DO, Mcclure LA, Soliman EZ, Judd $\mathrm{SE}$, et al. Differences in the role of black race and stroke risk factors for first vs recurrent stroke. Neurology 2016;86:637-42. https://doi.org/10.1212/WNL.0000000000002376

5.Tambara EM. Diretrizes para atendimento pré-hospitalar no acidente vascular encefálico. In: Cavalcante IL, Cantinho FAF, Assad AR (org.). Medicina Perioperatória. Rio de Janeiro:SAERJ, 2006, pp77-80.

6.Teixeira-Salmela LF, Olney SJ, Nadeau S, Brouwer B. Muscle strengthening and physical conditioning to reduce impairment and disability in chronic stroke survivors. Arch Phys Med Rehabil 1999;80:1211-8. https://doi.org/10.1016/s0003-9993(99)90018-7

7.Sharp SA, Brouwer BJ. Isokinetic strength training of the hemiparetic knee: Effects on function and spasticity. Arch Phys Med Rehabil 1997;78:1231-6. https://doi.org/10.1016/s0003-9993(97)90337-3

8. Polese JC, Mazzola D, Schuster RC. Eletroestimulação neuromuscular na pressão plantar, simetria e funcionalidade de hemiparéticos. Acta Fisiatr 2009;16:200-2.

https://www.revistas.usp.br/actafisiatrica/article/view/103288

9. Barcala L, Colella F, Araujo MC, Inoue AS, Oliveira CS. Análise do equilíbrio em pacientes hemiparéticos após o treino com o programa Wii Fit. Fisioter Mov 2011;24:337-43.

http://doi.org/10.1590/S0103-51502011000200015

10.Lacerda N, Gomes E, Pinheiro H. Efeitos da facilitação neuromuscular proprioceptiva na estabilidade postural e risco de quedas em pacientes com sequela de acidente vascular encefálico: estudo piloto. Rev Fisioter Pesq 2013;20:37-42. https://doi.org/10.1590/S1809-29502013000100007

11.Da Silva LRV, Rodacki ALF, Rebutini VZ, Góes SM, Coelho RW, Stefanello JMF. Efeitos de curto prazo de um programa de imaginação sobre o medo de queda de indivíduos pós acidente vascular encefálico. Motriz 2013;19:46-54. https://doi.org/10.1590/S198065742013000100005

12.Neves EB, Kruger E, Cióla MCS, Costin ACMS, Chiarello CR, Rosário MO. Terapia neuromotora intensiva na reabilitação da atrofia muscular 
espinhal: Estudo de caso. Rev Neurocienc 2014;22:66-74. https://doi.org/10.34024/rnc.2014.v22.8131

13.Scheeren EM, Mascarenhas LPG, Chiarello CR, Costin ACMS, Oliveira $L$, Neves EB. Description of the Pediasuit ProtocolTM. Fisioter Mov 2012;25:473-80. https://doi.org/10.1590/S0103$\underline{51502012000300002}$

14.Loffi RG, Guimarães, GR. Treini - Reeducação e Reabilitação Neurológica pelo Método Treini. (Acessado em 2021). Disponível em: www. metodotreini.com.br/quem-somos/

15.Fernandes BCW, Ferreira KCP, Marodin MF, Valmon FAR. Influência das orientações fisioterapêuticas na qualidade de vida e na sobrecarga de cuidadores. Fisioter Mov 2013;26:151-8.

https://doi.org/10.1590/S0103-51502013000100017

16.Cacho EWA, Melo FRLV, Oliveira R. Avaliação da recuperação motora de pacientes hemiplégicos através do protocolo de desempenho físico Fugl-Meyer. Rev Neurocienc 2004;12:94-102. https://doi.org/10.34024/rnc.2004.v12.8877

17. Hemingway $H$, Stephen MS, Shipley M, Marmot M. Is the SF-36 a valid measure of change in population health? Results from the Whitehall II Study. BMJ 1997;315:1273-9.

https://doi.org/10.1136/bmj.315.7118.1273

18.Smania N, Montagnana B, Faccioli S, Fiaschi A, Aglioti SM. Rehabilitation of somatic sensation and related deficit of motor control in patients with pure sensory stroke. Arch Phys Med Rehab 2003;84:1692-702. https://doi.org/10.1053/s0003-9993(03)00277-6 19.Scalzo P, Kummer A, Cardoso F, Teixeira AL. Depressive symptoms and perception of quality of life in Parkinson's disease. Arq. Neuropsiquiatr 2009;67:203-8. $\quad$ https://doi.org/10.1590/S0004282X2009000200006

20.Lawton M, Kleban M, Moss M, Rovine M, Glicksman A. Measuring caregiving appraisal. J Gerontol 1989;44:61-71.

https://doi.org/10.1093/geronj/44.3.p61

21.Jonsson AC, Lindgren I, Hallstrom B, Norrving B, Lindgren A. Determinants of quality of life in stroke survivors and their informal caregivers. Stroke 2005;36:803-8.

https://doi.org/10.1161/01.STR.0000160873.32791.20

22.Luna C, Dias LB, Santos SMS, Nunes LCBG. O Papel da Plasticidade Cerebral na Fisioterapia. Cerebro Mente 2002;15:13. https://cerebromente.org.br/n15/mente/plasticidade1.html 